\title{
Thalamic Innervation of the Direct and Indirect Basal Ganglia Pathways in the Rat: Ipsi- and Contralateral Projections
}

\author{
MARIA CASTLE, ${ }^{1}$ MARIA S. AYMERICH, ${ }^{2}$ CARLOS SANCHEZ-ESCOBAR, ${ }^{1}$ \\ NANCY GONZALO ${ }^{1}$ JOSÉ A. OBESO ${ }^{3}$ AND JOSÉ L. LANCIEGO ${ }^{1 *}$ \\ ${ }^{1}$ Department of Anatomy, Division of Neurosciences, Clínica Universitaria and Medical \\ School, Center for Applied Medical Research, University of Navarra, \\ 31008 Navarra, Spain \\ ${ }^{2}$ Department of Biochemistry, Division of Neurosciences, Clínica Universitaria and Medical \\ School, Center for Applied Medical Research, University of Navarra, \\ 31008 Navarra, Spain \\ ${ }^{3}$ Department of Neurology, Division of Neurosciences, Clínica Universitaria and Medical \\ School, Center for Applied Medical Research, University of Navarra, \\ 31008 Navarra, Spain
}

\begin{abstract}
The present study describes the thalamic innervation coming from the rat parafascicular nucleus (PF) onto striatal and subthalamic efferent neurons projecting either to the globus pallidus (GP) or to the substantia nigra pars reticulata (SNr) by using a protocol for multiple neuroanatomical tracing. Both striatofugal neurons targeting the ipsilateral $\mathrm{SNr}$ (direct pathway) as well as striatal efferent neurons projecting to the ipsilateral GP (indirect pathway) were located within the terminal fields of the thalamostriatal afferents. In the subthalamic nucleus (STN), both neurons projecting to ipsilateral GP as well as neurons projecting to ipsilateral SNr also appear to receive thalamic afferents. Although the projections linking the caudal intralaminar nuclei with the ipsilateral striatum and STN are far more prominent, we also noticed that thalamic axons could gain access to the contralateral STN. Furthermore, a small number of STN neurons were seen to project to both the contralateral GP and PF nuclei. These ipsi- and contralateral projections enable the caudal intralaminar nuclei to modulate the activity of both the direct and the indirect pathway. J. Comp. Neurol. 483:143-153, 2005. ๑ 2005 Wiley-Liss, Inc.
\end{abstract}

Indexing terms: striatum; globus pallidus; substantia nigra; parafascicular nucleus; neuroanatomy; Parkinson's disease

Current knowledge of basal ganglia circuitry has become more complex over the past few years. The "classical" basal ganglia model (Albin et al., 1989; DeLong, 1990) consists of two segregated feed-forward pathways, involving monosynaptic GABAergic projections from the striatum to the output nuclei (direct pathway), as well as a multisynaptic pathway successively linking the striatum with the external globus pallidus (GPe in primates, globus pallidus (GP) in rodents), the subthalamic nucleus (STN), and the output nuclei (known as the indirect pathway). Although this model is somewhat simplistic, it has led the basis for a reappraisal of surgical-based therapies in movement disorders (see Hamani et al., 2003, for a review). However, recent advances in physiological and anatomical research have provided more details of these circuits that must be integrated within the basal ganglia model (Bar-Gad and Bergman, 2001).

Grant sponsor: Michael J. Fox Foundation; Grant sponsor: Fondo de Investigaciones Sanitarias; Grant number: FIS 01/0237; Grant sponsor: Ministerio de Ciencia y Tecnología; Grant number: BFI2003-02033; Grant sponsor: Fondo Europeo de Desarrollo Regional; Grant sponsor: UTEproject/Foundation for Applied Medical Research.

*Correspondence to: José L. Lanciego, Neuroscience Division, Neuromorphology-tracing Lab, Center for Applied Medical Research, CIMA, 31008 Pamplona. Spain. E-mail: jlanciego@unav.es

Received 30 July 2004; Revised 3 September 2004; Accepted 14 October 2004

DOI 10.1002/cne.20421

Published online in Wiley InterScience (www.interscience.wiley.com). 
First, there is overwhelming evidence that the basal ganglia circuits are highly collateralized. Efferent neurons within the striatum, both segments of the globus pallidus, STN, and the substantia nigra pars reticulata (SNr) interact with different target structures through axon collaterals (see Parent et al., 2000, for a review). Second, the $\mathrm{GPe}$ can no longer be seen as a simple relay nucleus located between the striatum and the STN. Hence, what was formerly known as an "indirect pathway" is currently considered as a complex network in its own right, where the STN and both the internal and external divisions of the globus pallidus (GPi and GPe, respectively) are closely interconnected, modulating basal ganglia output (Shink et al., 1996; Joel and Weiner, 1997; Nambu, 2004). Finally, the roles of transverse circuits that link the thalamus to the striatum and STN have been largely neglected in most studies dealing with basal ganglia function (Obeso et al., 2000). Besides cortical inputs, the rat parafascicular thalamic nucleus (PF) is known to be one of the most important sources of striatal afferents (Féger et al., 1994; Deschênes et al., 1995, 1996; Rudkin and Sadikot, 1999; Gonzalo et al., 2002; Vercelli et al., 2003). Within the striatum, projection neurons are approached by thalamic afferents (Dubé et al., 1988), resulting in an excitatory effect (Buchwald et al., 1973; Kitai et al., 1976; Kocsis et al., 1977; Wilson et al., 1983). Indeed, it is worth noting that different types of striatal interneurons are known to be postsynaptic targets for thalamostriatal afferents (Lapper and Bolam, 1992; Bennet and Bolam, 1994; Sidibé and Smith, 1999; Rudkin and Sadikot, 1999). Thalamic afferents arising from the PF may also have access to the STN (Marini et al., 1999; Gonzalo et al., 2002), these projections being arranged topographically (Lanciego et al., 2004). Moreover, thalamo-subthalamic projections are mediated by glutamate (Mouroux and Féger, 1993; Mouroux et al., 1995) and are known to be overactive in unilaterally dopamine-depleted rats (Orieux et al., 2000). This issue raises the question of whether the increased activity of the STN upon unilateral dopamine depletion might be provoked by thalamic overactivity. In this regard, Bacci et al. (2004) recently demonstrated that the ibotenateinduced lesion of the caudal intralaminar nuclei is highly effective in preventing the increases in metabolic activity within the STN, GP, SNr, and entopeduncular nucleus (ENT, the rodent analog of primate GPi) that typically follow unilateral dopamine depletion in rats.

In order to assess the potential roles of thalamic neurons in the modulation of basal ganglia transmission at different levels, we performed a multiple tracing study

\section{Abbreviations}

$\begin{array}{ll}\text { BDA } & \text { Biotinylated dextran amine } \\ \text { CLSM } & \text { Confocal laser scanning microscope } \\ \text { CM } & \text { Centromedian thalamic nucleus } \\ \text { CM-PF } & \text { Centromedian-parafascicular thalamic complex } \\ \text { CTB } & \text { Cholera toxin, } \beta \text { subunit } \\ \text { ENT } & \text { Entopeduncular nucleus } \\ \text { FG } & \text { Fluoro-Gold } \\ \text { GP } & \text { Globus pallidus } \\ \text { GPe } & \text { Globus pallidus, external division } \\ \text { GPi } & \text { Globus pallidus, internal division } \\ \text { PF } & \text { Parafascicular thalamic nucleus } \\ \text { SNr } & \text { Substantia nigra reticulata } \\ \text { STN } & \text { Subthalamic nucleus }\end{array}$

based on confocal laser-scanning microscopy (CLSM). Our aim was to elucidate whether the striatal and STN neurons that project to the same area of the GP receive thalamic inputs from the PF, and whether PF neurons innervate striatal and STN neurons projecting to the same area of the $\mathrm{SNr}$.

\section{MATERIALS AND METHODS}

A total of six female Wistar rats (240-290 g) were used in this study. At all times animals were handled according to the European Council Directive 86/609/EEC as well as in agreement with the Society for Neuroscience Policy on the Use of Animals in Neuroscience Research. The experimental design was approved by the Ethical Committee for Animal Testing at the University of Navarra (Ref. 037/2000).

\section{Surgical procedure}

Animals were deeply anesthetized with an intraperitoneal injection of a mixture of four parts of Ketaset (1\% of a solution of ketamine, $75 \mathrm{mg} / \mathrm{kg}$ ) and three parts of Rompun ( $2 \%$ of a solution of xylazine, $10 \mathrm{mg} / \mathrm{kg}$ ). The rats were then placed in a stereotaxic frame (David Kopf, Tujunga, CA; frame for small animals) and all tracers were injected in a single surgical session. The stereotaxic coordinates were taken from the atlas of Paxinos and Watson (1998). The tracer biotinylated dextran amine (BDA, Molecular Probes Europe, Leiden, The Netherlands) was iontophoretically delivered to the left parafascicular nucleus $(\mathrm{PF})$ in a $10 \%$ solution in $0.01 \mathrm{M}$ phosphate buffer (PB), $\mathrm{pH}$ 7.25 , using a glass micropipette (inner tip diameter $25-40$ $\mu \mathrm{m}$ ) and a positive-pulsed direct current ( $5 \mu \mathrm{A}, 7$ seconds on/off). In order deposit only small amounts, the tracer was injected for $\sim 3-5$ minutes and once completed the micropipette was left in place for 10 minutes before withdrawal. During removal of the micropipette the polarity of the current was reversed in order to minimize tracer uptake through the injection tract. Next, the tracer cholera toxin $\beta$ subunit (CTB, List Biological Laboratories, Campbell, CA) was pressure-injected $(0.5 \mu \mathrm{l}$ of a $2 \%$ solution in $0.1 \mathrm{M} \mathrm{PB}, \mathrm{pH} 7.3$ ) with a Hamilton syringe in the ipsilateral substantia nigra reticulata $(\mathrm{SNr})$. Finally, the retrograde tracer Fluoro-Gold (FG, Fluorochrome LLC, Denver, $\mathrm{CO}$ ) was iontophoretically delivered in the ipsilateral globus pallidus (GP) in a $2 \%$ solution in $0.1 \mathrm{M}$ cacodylate buffer, pH 7.3, using the same parameters as described for the BDA injections.

\section{Tissue processing}

One week postinjection, animals were anesthetized with an overdose of $10 \%$ chloral hydrate and then transcardially perfused with a saline Ringer's solution followed by $500 \mathrm{~mL}$ of a cold fixative solution containing $4 \%$ paraformaldehyde and $0.1 \%$ glutaraldehyde in $0.125 \mathrm{M} \mathrm{PB}, \mathrm{pH}$ 7.4. After perfusion, the skull was opened and the brain removed and stored in a cryoprotective solution containing $20 \%$ glycerin and $2 \%$ dimethylsulphoxide in $0.125 \mathrm{M}$ PB, pH 7.4 (Rosene et al., 1986). Frozen coronal microtome sections $(40-\mu \mathrm{m})$ were obtained and collected in $0.125 \mathrm{M}$ $\mathrm{PB}, \mathrm{pH} 7.4$, in 10 series of adjacent sections. Every tenth section was used for triple neuroanatomical tracing combining BDA, CTB, and FG. The remaining series were stored $\left(-80^{\circ} \mathrm{C}\right)$ until used for further histological processing. 


\section{Histology}

The sections were first incubated in a cocktail of primary antisera containing goat anti-CTB $(1: 2,000$; List Biological) and rabbit anti-FG (1:2,000; Chemicon, Temecula, CA) for 60 hours at $4^{\circ} \mathrm{C}$. Next, the sections were exposed to a cocktail of bridge antisera containing donkey antigoat IgG labeled with Alexa 546 (1:50; Molecular Probes) and donkey antirabbit IgG labeled with Alexa 488 (1:50; Molecular Probes) for 2 hours at room temperature. Finally, sections were incubated in streptavidin labeled with Alexa 633 (1:100) for 90 minutes at room temperature.

Sections were mounted on glass slides using a $0.2 \%$ solution of gelatin (Merck, Darmstadt, Germany) in 0.05 $\mathrm{M}$ Tris/HCl pH 7.6, dried at room temperature in the dark, rapidly dehydrated in toluene, and coverslipped with Entellan (Merck). All antisera used in this procedure were diluted in $0.05 \mathrm{M}$ Tris-buffered-saline, $\mathrm{pH} 8$, with $0.5 \%$ Triton X-100 (Sigma, St. Louis, MO; TBS-Tx). The incubations in the primary antisera were performed with $2 \%$ bovine serum albumin (BSA, Merck). Extensive washing with $0.05 \mathrm{M}$ TBS-Tx, $\mathrm{pH} 8$, was carried out throughout the procedure. Several rinses with $0.05 \mathrm{M}$ Tris/HCl, $\mathrm{pH} 7.6$, were conducted prior to mounting the sections in gelatin.

\section{Quantitative analysis}

In order to obtain an overall estimate of the number of double-labeled cells within the striatum and STN, a semiquantitative analysis of labeled neurons was performed in six different animals in the $\mathrm{X}-$, Y-, and Z-axis using CLSM. This was carried out by taking four snapshots at a magnification of $40 \times$ from the dorsal, lateral, ventral, and medial areas of the striatum. The analysis comprised six striatal rostrocaudal levels (four precommissural and two postcommissural sections), equally spaced $400 \mu \mathrm{m}$ from each other. Neurons positive for FG ("green" channel) were counted first, followed by the CTB-labeled neurons in the "blue" channel. Subsequently, the double-labeled neurons were unequivocally identified in the "overlay" channel. A similar analysis was also carried in the STN, where three snapshots were taken from medial, central, and lateral STN territories, these measurements involving three rostrocaudal STN levels.

\section{Confocal visualization settings}

The analysis was conducted using a Zeiss confocal microscope (LSM 510 Meta) equipped with an argon laser with excitation wavelengths of $458,477,488$, and $514 \mathrm{~nm}$, one helium-neon laser with an excitation wavelength of $543 \mathrm{~nm}$, and another helium-neon laser with an excitation wavelength of $633 \mathrm{~nm}$. In order to ensure the appropriate visualization of labeled elements and to avoid falsepositive results, the emission from the argon laser at 488 $\mathrm{nm}$ was filtered through a bandpass filter of $505-530 \mathrm{~nm}$, this emission being color-coded in green. The emission following the excitation with the helium laser at 543 was filtered through a bandpass filter of $560-615 \mathrm{~nm}$, colorcoded in light blue. Finally, a longpass filter of $650 \mathrm{~nm}$ was used for visualization of the emission from the helium laser at $633 \mathrm{~nm}$, this emission being color-coded in red. The sections were first examined using low-magnification lenses $(\times 10$ and $\times 20)$ and photographs were then taken at higher magnification $(\times 40$ oil-immersion objective, NA 1.3 , and $\times 63$ oil-immersion objective, NA 1.4 ).

\section{RESULTS}

The data we report here were obtained from the analysis of six cases from a total of 38 animals that underwent stereotaxic surgery. Only those animals in which the three injected tracers were confined to their respective target area were considered as valid for this study. Animals in which the tracer had leaked into the injection tract were rejected. A schematic representation of the injection sites is shown in Figure 1.

\section{Ipsilateral thalamostrial projections and their relationship with identified subpopulations of striatal neurons}

The delivery of retrograde tracers to either the GP or $\mathrm{SNr}$ (FG and CTB, respectively) led to a large number of neurons being labeled throughout the entire rostrocaudal extent of the striatum (Fig. 2). Three different neuronal populations were observed: 1) the most numerous population consisted of striatal neurons projecting only to the GP (single-labeled with FG), these comprising 54.5\% of the total amount of labeled cells; 2) striatonigral neurons single-labeled with CTB (18.41\%); and 3) striatal neurons projecting to both the GP and SNr, double-labeled with FG and CTB $(27.2 \%)$. Of the striatal cells that were labeled with FG after GP injection, an average of $34.3 \%$ were double-labeled with CTB. As such, up to a third of the striatal efferent cells projecting to the GP have collaterals that project to the SNr. Approximately 59.6\% of the striatal cells that were labeled with CTB were also labeled with FG, suggesting that the majority of striatonigral neurons send collaterals that terminate in the GP. The distribution of the labeled neurons within the striatum depended on the location of the injection sites in the GP or SNr. Striatopallidal neurons projecting to dorsomedial, dorsolateral, ventromedial, and ventrolateral GP areas were located in the dorsomedial, dorsolateral, ventromedial, and ventrolateral striatal territories, respectively. Striatonigral neurons were also found throughout the preand postcommissural striatum. Neurons located in the medial and ventral striatal regions preferentially innervated medial $\mathrm{SNr}$ areas, while lateral $\mathrm{SNr}$ areas were innervated by striatal neurons located in dorsal and lateral striatal territories. In all cases, double-labeled neurons projecting to both the GP and SNr were found intermingled with single-labeled cells, without presenting any preferred location in the striatum.

Injection of BDA into the $\mathrm{PF}$ resulted in intense anterograde labeling of thin varicose fibers that spread throughout the entire rostrocaudal extent of the striatum. In general, thalamostriatal fibers were aggregated in patches or bands of terminal arborizations. Their distribution varied, depending on the site of injection in the $\mathrm{PF}$, as reported previously (Lanciego et al., 2004). Striatal neurons projecting to GP as well as striatal neurons projecting to $\mathrm{SNr}$ were often located within patches of anterogradely labeled thalamic afferents (Fig. 2).

\section{Ipsilateral thalamo-subthalamic projections and their relationship with STN efferent neurons}

Massive retrograde cell labeling was observed in the STN as a result of tracer deposits in either the GP or SNr. In agreement with earlier reports (Van der Kooy and Hattori, 1980; Kita et al., 1983; Kita and Kitai, 1987; 


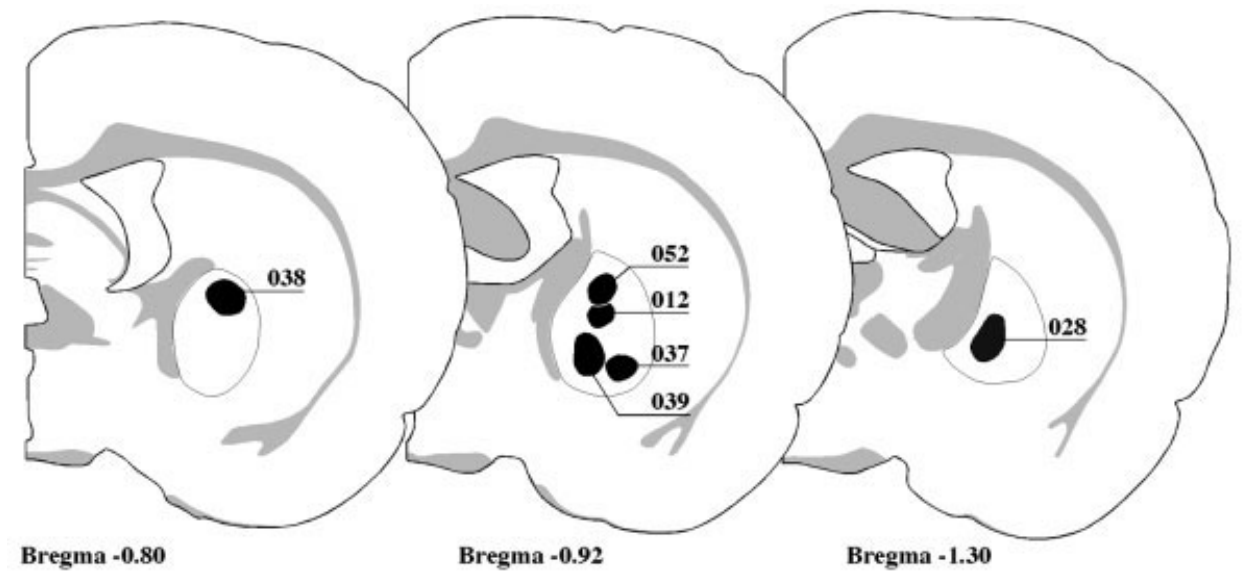

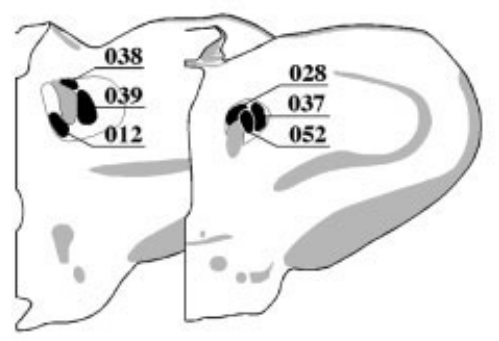

Bregma -4.16 Bregma -4.30

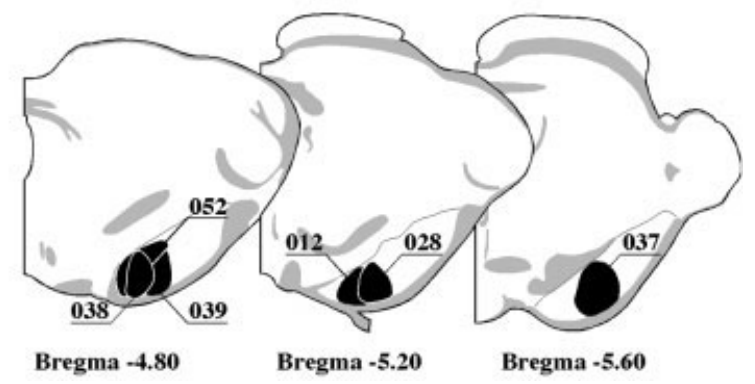

Fig. 1. Flow chart illustrating the localization and extent of the injection sites of the cases analyzed in the current study. Top: Fluoro-Gold deposits in the globus pallidus. Bottom: Injection sites of the anterograde tracer biotinylated dextran amine (left) and the retrograde tracer cholera toxin $\beta$ subunit (right) within the rat parafascicular nucleus and substantia nigra reticulata, respectively.
Plenz and Kitai, 1987; Parent et al., 2000), efferent STN axons are highly branched, the most prominent cell types were double-labeled neurons with FG and CTB. Hence, most of the efferent STN neurons, $66.4 \%$ of the total amount of labeled neurons, projected to both GP and SNr. Neurons that projected only to the GP (single-labeled with FG) represented $20.4 \%$ of the labeled neurons on average, while a smaller population of STN neurons projected exclusively to the $\mathrm{SNr}$ (10.2\% of CTB-single-labeled neurons). Of the STN cells labeled with FG after injection to the GP, an average of $76.4 \%$ were double-labeled with CTB. This indicates that a large number of STN efferent cells projecting to the GP have collaterals to the $\mathrm{SNr}$. Indeed, $\sim 86.6 \%$ of the STN cells labeled with CTB were double-labeled with FG, suggesting that the majority of STN neurons projecting to the $\mathrm{SNr}$ send collaterals that terminate in the GP. Both the FG- and CTB-labeled neurons were intermingled, and they were distributed throughout the entire extent of extent of the STN nucleus, with no single preferred location (Fig. 3).

Thalamic axons arising from the $\mathrm{PF}$ arborized profusely within the STN and these thalamic afferents consisted of thin, densely branched, varicose fibers. Both single- and double-labeled STN efferent neurons were found within terminal fields of thalamic afferents (Fig. 3). Although the distal dendrites of STN neurons are known to be the main target for thalamic glutamatergic axons, several axosomatic contacts between thalamic afferents and STN neurons projecting to the GP were clearly identified (Fig. 3).

The anterograde tracer BDA can also be transported bidirectionally, often resulting in Golgi-like labeling. Accordingly, a reduced number of neurons retrogradely la- beled with BDA were observed within the STN, representing a small population of STN neurons that projected to the ipsilateral PF (up to $2.2 \%$ of the total amount of STN retrogradely labeled neurons; Fig. 3) These neurons were single-labeled with BDA and appeared to be approached by thalamic inputs.

\section{Contralateral thalamo-subthalamic projections}

In all animals injected with BDA in PF, a small number of anterograde labeled fibers were observed within the contralateral STN. These fibers reached the STN laterally, arborizing according to a complex pattern in medial STN areas. The terminal fields of contralateral thalamo-subthalamic axons were typically characterized by thick varicosities and a high density of terminal boutons (Fig. 4).

\section{Contralateral subthalamo-pallidal and subthalamo-thalamic projections}

Due to the bidirectional nature of the tracer BDA, a small number of retrograde labeled neurons were also observed in the contralateral STN (2-5 neurons per section). As such, there was a small subpopulation of efferent STN neurons that projected directly to the contralateral $\mathrm{PF}$, in accordance with the pioneering observations made by Gerfen et al. (1982). Furthermore, these BDA-labeled neurons also contained retrograde transported FG (delivered to the contralateral GP), indicating that these double-labeled neurons project to both the contralateral $\mathrm{PF}$ and GPe (Fig. 5). This phenotype of double-labeled 

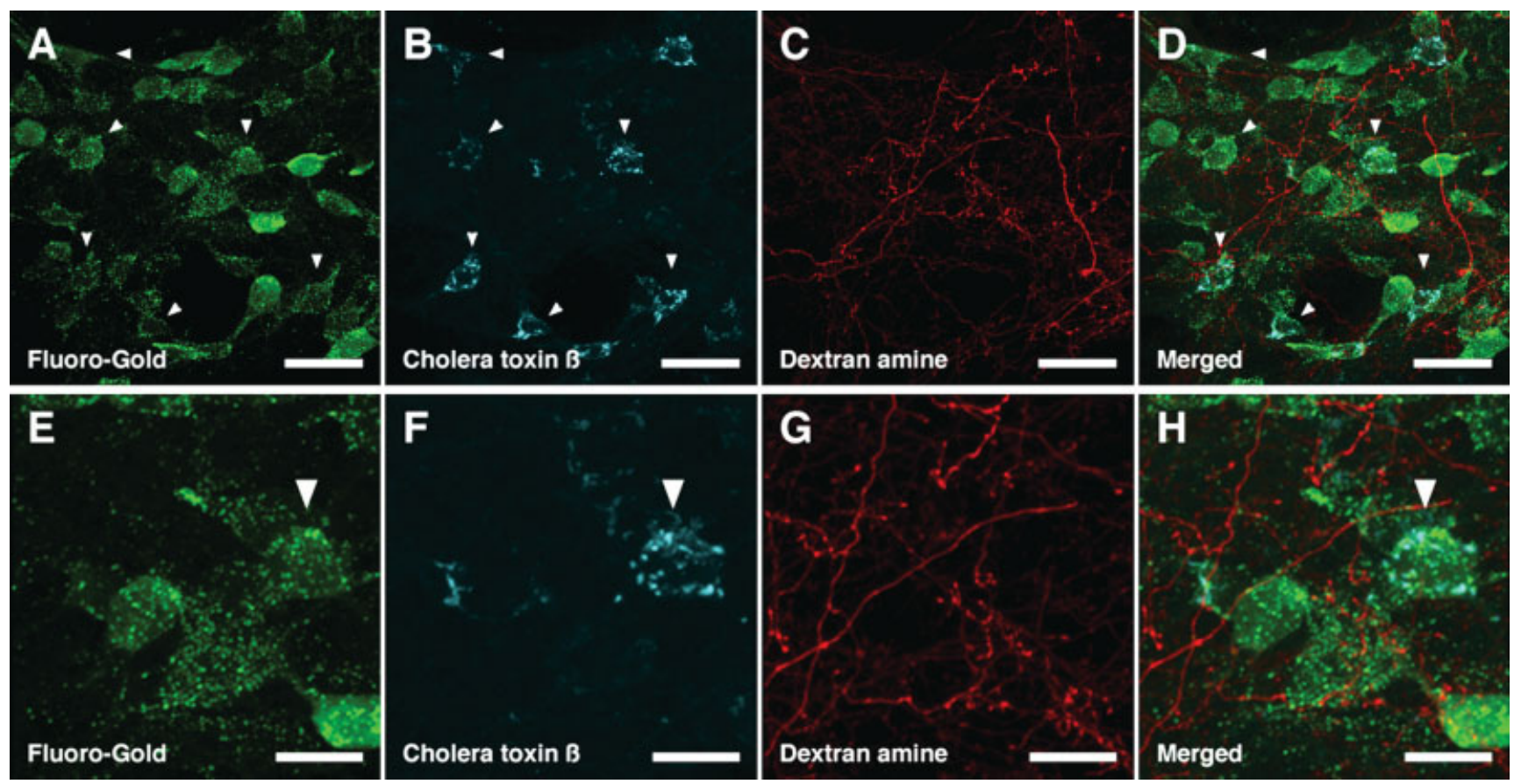

Fig. 2. Thalamostriatal projections and their relationships with identified ipsilateral striatofugal neurons. A-D: Medium-power photomicrographs taken from coronal sections through the precommissural striatum (case 028) showing retrograde tracing with FG (A) and CTB (B), corresponding to striatopallidal and striatonigral neurons, respectively, as well as anterograde tracing with BDA coming from

the PF (C). White arrowheads indicate double-labeled cells. D: Threechannels overlay showing striatal double-labeled cells (white arrowheads) as well as the relationships between thalamic afferents and striatal projection neurons. E-H: Insets are higher magnification images of one double-labeled neuron (white arrowhead). Scale bars = $60 \mu \mathrm{m}$ in $\mathrm{A}-\mathrm{D} ; 30 \mu \mathrm{m}$ in $\mathrm{E}-\mathrm{H}$

STN efferent cells was seen in all cases, independent of the location of the tracer deposits within the GP and PF.

\section{DISCUSSION}

The present study provides evidence of the complex modulation of basal ganglia circuits by thalamic inputs arising from the caudal intralaminar nuclei in rats. At the striatal level, neurons projecting to the GP, neurons projecting to the $\mathrm{SNr}$, and neurons projecting to both the GP and SNr are located within the terminal fields of thalamostriatal afferents. Furthermore, up to five distinct types of efferent neurons were found in the STN: 1) neurons projecting to the GP; 2) neurons projecting to the $\mathrm{SNr} ; 3$ ) neurons projecting to both the GP and SNr; 4) neurons projecting to the ipsilateral $\mathrm{PF}$; and 5) neurons projecting to both the contralateral PF and GP. Finally, it is worth noting that the STN receives two different kinds of thalamic innervations, a massive projection from the ipsilateral $\mathrm{PF}$, and a more sparse projection originating in the contralateral caudal intralaminar nuclei. A flow-chart illustrating a summary of the labeled elements observed here is provided in Figure 6.

\section{Technical considerations}

With respect to the possible appearance of staining artifacts, some methodological considerations should be taken into account. First of all, the incorrect detection of fluorescent emissions by the CLSM might naturally led to the detection of a false signal. The use of bandpass filters (as stipulated in Materials and Methods) together with the use of the so-called "multitracking" scanning in the $\mathrm{X}-\mathrm{Y}-\mathrm{Z}$ axis ensures the appropriate visualization of the fluorescent emissions from each fluorescent dye. In this way, we have avoided the so-called phenomenon of "crosstalk." Another possible source of false signals is related to the uptake of FG by fibers of passage, since in rodents the GP is an area rich in fibers. It is generally accepted that by performing iontophoretical delivery of the dye the amount of FG that is taken up by fibers damaged in the injection process is minimized (Schmued and Fallon, 1986; Pieribone and Aston-Jones, 1988; Schmued and Heimer, 1990; Divac and Mogensen, 1990).

A further limitation of the methods we have applied is the difficulty in defining whether a physical contact represents the presence of a synapse at the light microscopical level. While the CLSM increases the resolution by a factor of 1.4 in comparison to light microscopy (Pawley, 1995; Inoué, 1995), terminal boutons or synaptic contacts can only be properly visualized with electron microscopy. Nevertheless, we have followed the criteria suggested by Wouterlood et al. (2002a,b) to ascertain whether a point of physical contract represents a "true" synapse. However, the accurate visualization of synaptic contacts between labeled fibers and dendritic shafts or spines located far away from the neuron soma is sometimes hampered by the intrinsic nature of retrograde tracing techniques. Indeed, even with sensitive retrograde tracers such as FG, the transported marker is most often only visible within the neuronal soma and the thickest main dendrites. 

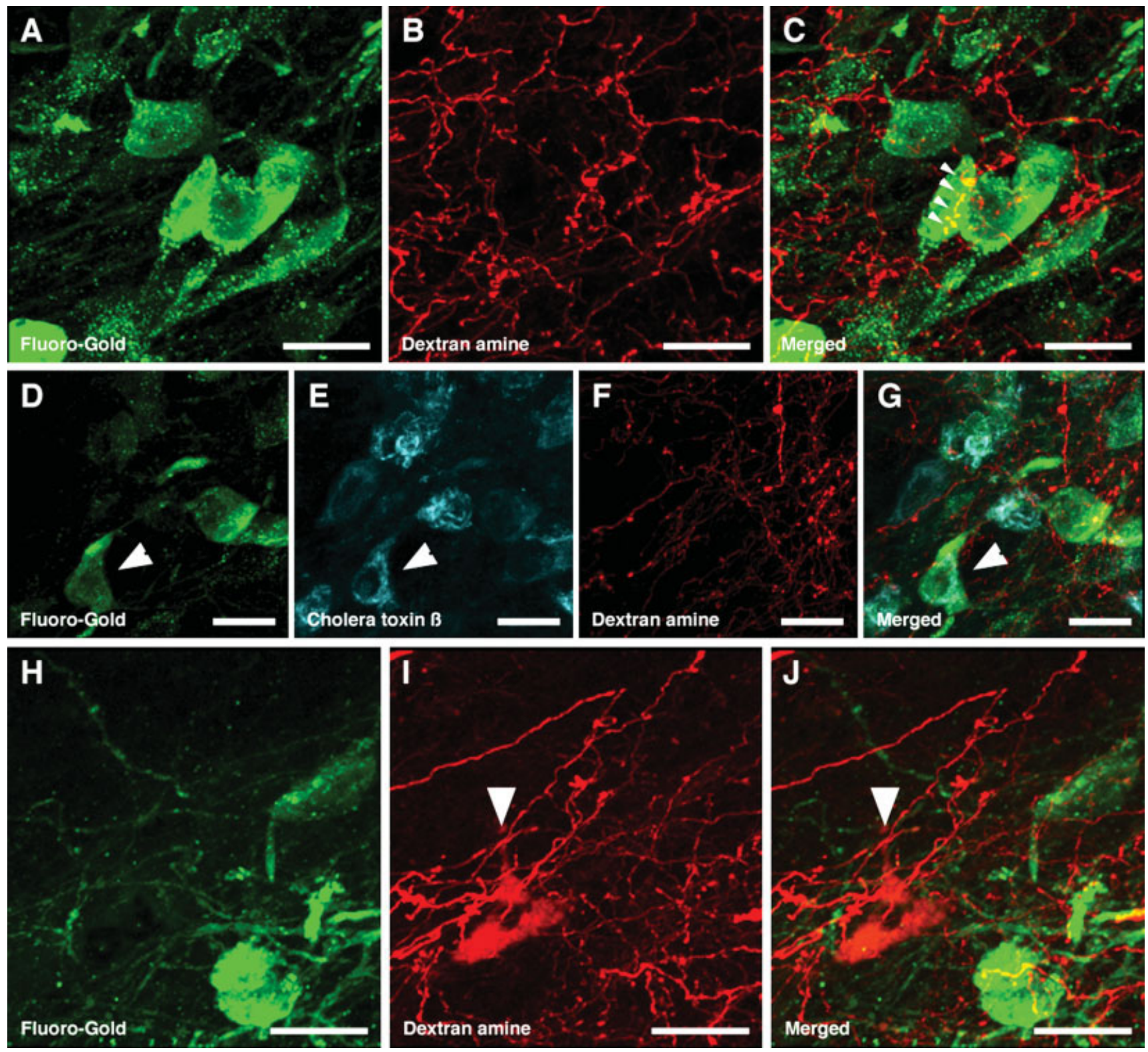

Fig. 3. Thalamosubthalamic projections and their relationships with identified ipsilateral STN efferent neurons. A-C: Case 037. Dual detection of FG and BDA. Microphotographs taken from coronal sections through the STN showing FG-labeled neurons (color-coded in green) projecting to the ipsilateral GP. These neurons are afferented by thalamic axons (color-coded in red). BDA afferents to the STN are distributed in patches comprised of densely branched fibers containing numerous varicosities and terminals. White arrowheads indicate several axosomatic contacts. D-G: Case 039. Triple detection of FG, CTB, and BDA (color-coded in green, blue, and red, respectively). STN

efferent neurons projecting to the $\mathrm{GP}$ or $\mathrm{SNr}$ were retrogradely labeled with either FG or CTB, respectively. As an example, a doublelabeled neuron is indicated by a white arrowhead. H-J: Ipsilateral subthalamothalamic projections. Dual detection of FG (color-coded in green) and BDA (color coded in red). Case 012. One BDA-containing neuron is visible in the red channel as well as in the overlay (white arrowheads). This neuron is retrogradely labeled after deposition of BDA in the ipsilateral PF. Several close appositions between the labeled neuron and thalamic afferent fibers were seen. Scale bars $=$ $40 \mu \mathrm{m}$.

\section{Collateral projections of striatofugal and subthalamofugal neurons}

Striatal efferent neurons with axons that reach the GP are known to express the peptide enkephalin (ENK-ir), whereas those neurons with axons terminating in the $\mathrm{SNr}$ coexpress substance P (SP-ir) and dynorphin (Anderson

and Reiner, 1990; Reiner and Anderson, 1990). Furthermore, results from in situ hybridization studies suggest that SP-ir neurons express D1 receptor mRNA, but little or no D2 transcripts, and that ENK-ir neurons express D2 receptor transcripts, with little or no D1 receptor mRNA (Gerfen et al., 1990; Le Moine et al., 1991; Gerfen, 1992; Robertson, 1992). These results have established the basis 

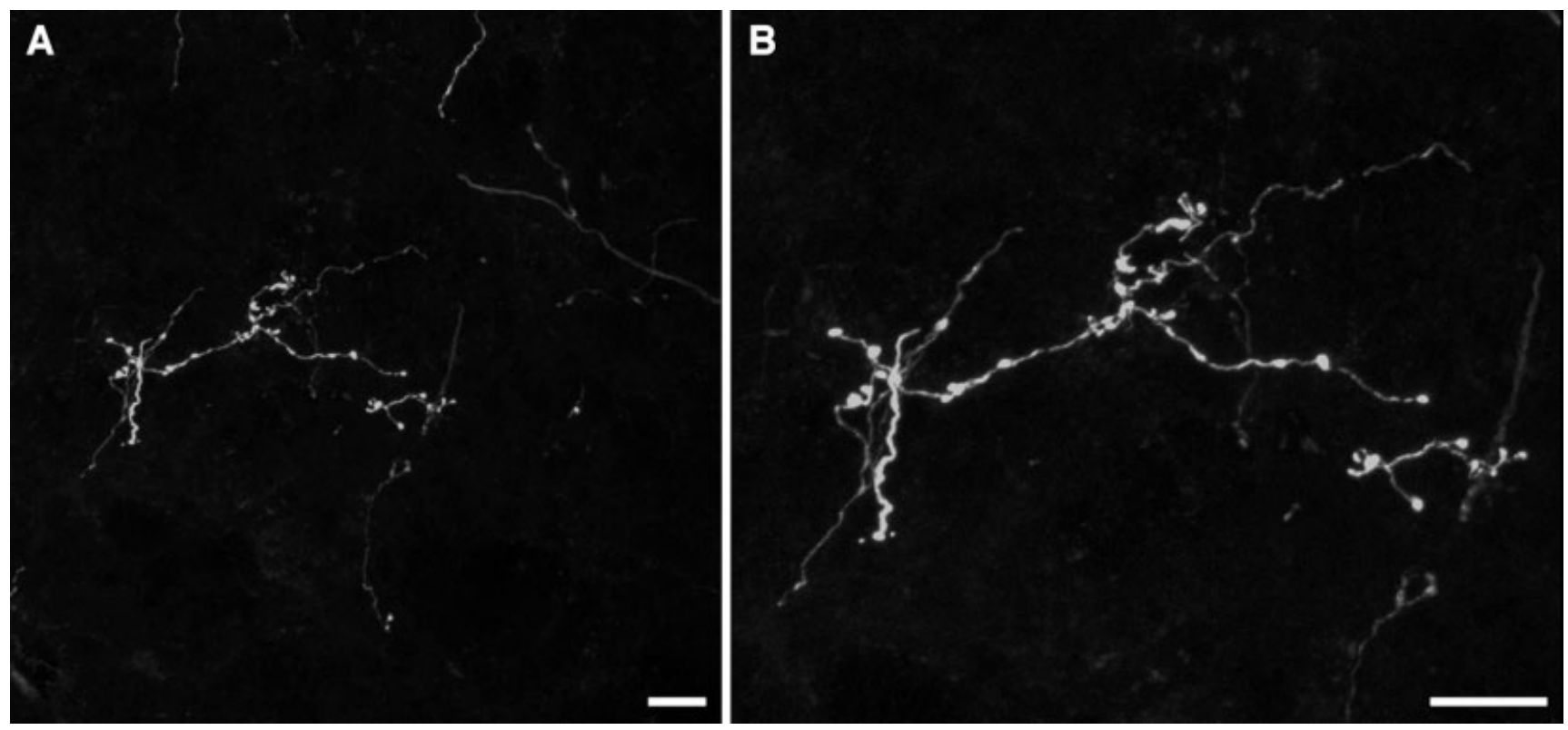

Fig. 4. Contralateral thalamosubthalamic projections. Case 037. Medium power photomicrograph (A) and inset at higher magnification (B) taken from the STN nucleus contralateral to the PF nucleus injected with BDA. Crossed thalamosubthalamic projections involve a reduced number of profusely branched fibers, all of them containing thick varicosities and terminal boutons. Scale bars $=45 \mu \mathrm{m}$.

for the segregation of the striatal output pathways, one of the cornerstones of the current basal ganglia model. However, other studies have challenged these conclusions, showing a considerable overlap (25-42\%) in the expression of D1 and D2 receptor mRNA in striatal neurons (Meador-Woodruff et al., 1991; Weiner et al., 1991). Indeed, Surmeier et al. $(1992,1993)$ showed that up to $60 \%$ of striatonigral neurons contain D2 protein and, more recently, both receptor subtypes were identified in retrogradely labeled striatonigral and striatopallidal neurons using CLSM analysis of fluorescent tagged D1A and D2L receptor antibodies (Ariano et al., 1993). Sensitive singlecell labeling studies in rats and primates have revealed that the striatofugal system is highly collateralized, about two-thirds of striatofugal neurons project to two or three striatal target structures (Kawaguchi et al., 1990; Parent et al., 1995, 2000; Wu et al., 2000). Up to three different types of striatofugal neurons were identified: type I neurons that project only to the GP; type II neurons that project to both the GP and SNr; and type III neurons that reach the GP, the entopeduncular nucleus, and the $\mathrm{SNr}$ (for more details, see Wu et al., 2000). Thus, the striatofugal system cannot longer be seen as a projection system characterized by a simple mode of dual transmission (direct and indirect pathways). Our results confirm the existence of collateral projections from striatofugal neurons, showing that an average of $34.3 \%$ of striatopallidal neurons also project to the $\mathrm{SNr}$, while the majority of striatonigral neurons $(59.6 \%)$ send collaterals to terminate in GP. Some minor differences between the data from juxtacellular studies and that obtained using retrograde tracers are likely to reflect the lack of concordance between the location of the injection sites and that of the pallidal and nigral axonal arborization of striatofugal neurons.

The highly branched nature of STN axons has been confirmed in several anatomical studies indicating that most STN neurons send axons to both the SNr and GPi (Van der Kooy and Hattori, 1980; Kita et al., 1983; Kita and Kitai, 1987; Plenz and Kitai, 1999). More recent anatomical data identified up to five distinct subtypes of STN efferent neurons each of these subtypes being characterized by a distinctive pattern of axonal collateralization (Sato et al., 2000; Parent et al., 2000). In agreement with these reports, our results show that an average of $76.4 \%$ of subthalamopallidal neurons also project to the $\mathrm{SNr}$, while most subthalamonigral neurons send collaterals towards the GP $(86.6 \%)$.

In summary, the data presented here are in accordance with earlier reports showing that both the striatofugal and subthalamofugal projections are highly collateralized systems, whose elements are endowed with highly branched axons enabling them to simultaneously influence several basal ganglia nuclei. At this point, it is worth noting that all types of striatal and subthalamic efferent neurons seemed to be tentatively approached by thalamic afferents, as discussed below.

\section{Ipsilateral thalamic modulation of basal ganglia circuits}

The role of the caudal intralaminar nuclei in basal ganglia function has generated interest over the past few years. Significant cell loss has been found in the centromedian-parafascicular complex (CM-PF, the analog to the rodent $\mathrm{PF}$ in higher mammals) in the brains of patients with Parkinson's disease (Henderson et al., $2000 a, b)$. Furthermore, the ibotenate-induced lesion of the $\mathrm{PF}$ in healthy rats results in dramatic changes in the metabolic activity of several basal ganglia nuclei, including the striatum, the output nuclei, and intrinsic nuclei such as the GP and STN (Bacci et al., 2002). When considering unilaterally dopamine-depleted rats, the chemical lesion of $\mathrm{PF}$ is capable of preventing the increase in 

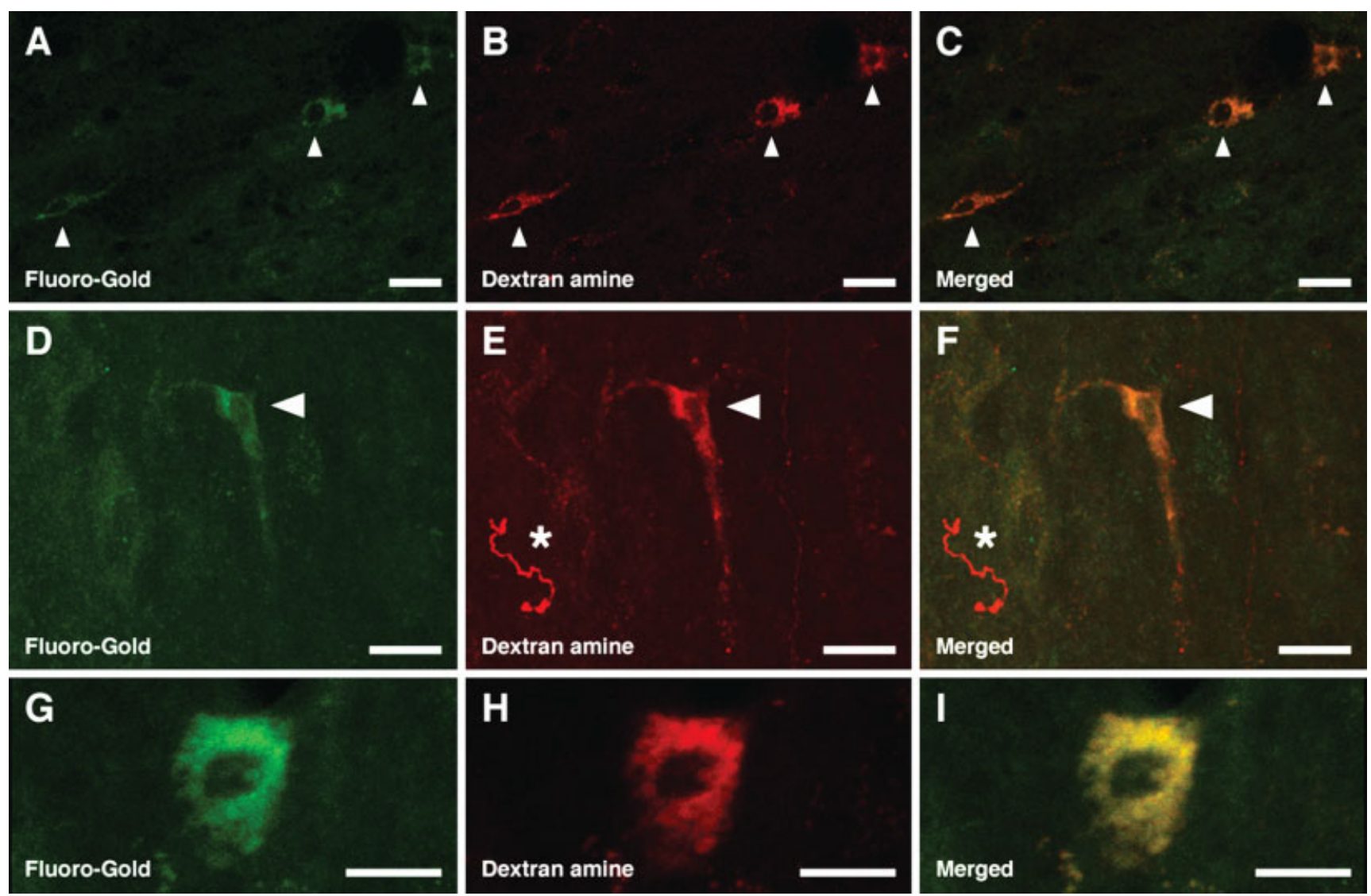

Fig. 5. STN neurons giving rise to contralateral subthalamothalamic and subthalamopallidal projections. Low- (A-C), medium- (DF), and high-magnification photomicrographs (G-I) illustrating STN efferent neurons, double-labeled with both BDA (contralateral subthalamothalamic projecting neurons) and FG (contralateral sub-

thalamopallidal projecting neurons). Examples are taken from cases 012 (A-C), 028 (D-F), and 037 (G-I). Arrowheads indicate the doublelabeled cells. One single BDA-labeled fiber, coming from the contralateral PF, is also visible (asterisk in E,F). Scale bars $=40 \mu \mathrm{m}$ in A-F; $30 \mu \mathrm{m}$ in $\mathrm{G}-\mathrm{I}$. metabolic activity typically observed in the output nuclei (Bacci et al., 2004).

At the striatal level, the postsynaptic structures reached by neurons from the caudal intralaminar nuclei include several types of striatal interneurons (Lapper and Bolam, 1992; Bennet and Bolam, 1994; Rudkin and Sadikot, 1999; Sidibé and Smith, 1999), as well as striatal neurons projecting to the output nuclei (Lanciego et al., 2004). According to the data reported here, the fact that $\mathrm{PF}$ fibers appear to contact striatal neurons involved in either the direct or indirect pathways contrast with findings in monkeys where striatal neurons projecting to the GPi are preferentially afferented by thalamic axons (Sibidé and Smith, 1996). We consider that this variation does not reflect a major difference between rodents and primates in the organization of the thalamostriatal system, but simply reflects the different methodological approach used in the two studies.

The thalamus is thought to modulate the activity of the GP and $\mathrm{SNr}$ in a highly complex manner. On the one hand, neurons located in the caudal intralaminar nuclei supply afferents to both striatal and subthalamic neurons projecting to the same area of the GP. Thalamic projections to striatopallidal neurons might reduce the activity of GP neurons by increasing the amount of GABA re- ceived by pallidal neurons. In contrast, the excitatory thalamic innervation of glutamatergic subthalamopallidal neurons might induce the opposite effect. On the other hand, PF neurons also innervate striatal and STN neurons projecting to a single area of the SNr. Therefore, the resulting activity of $\mathrm{SNr}$ neurons might be seen as a balance of increased GABA innervation coming from striatonigral neurons receiving thalamic excitation, and increased glutamate levels coming from subthalamonigral-projecting neurons, which also receive thalamic afferents. Besides the role played by thalamo-striato-pallidal, thalamo-subthalamo-pallidal, thalamo-striato-nigral, and thalamo-subthalamo-nigral loops, it is also worth noting that direct $\mathrm{PF}$ projections to the entopeduncular nucleus, $\mathrm{SNr}$, and GP have been reported elsewhere (Kincaid et al., 1991; Marini et al., 1999). In this regard, recent anatomical work by Yasukawa et al. (2004) nicely demonstrated that PF afferents to the GP are topographically organized in parallel to the PF projections to the striatum.

\section{Anatomical substrates underlying a complex thalamic modulation of STN activity}

The increased activity of the STN is thought to exert an important influence on basal ganglia pathophysiology, al- 
Fig. 6. Flow chart summarizing the main findings of the present study. The complex arrangement of the $\mathrm{PF}$ efferent pathways, together with the existence of ipsi- and contralateral projections, enables the PF nucleus to exert a multifaceted influence on basal ganglia circuits, at different levels. The caudate-putamen is abbreviated $\mathrm{CPu}$.

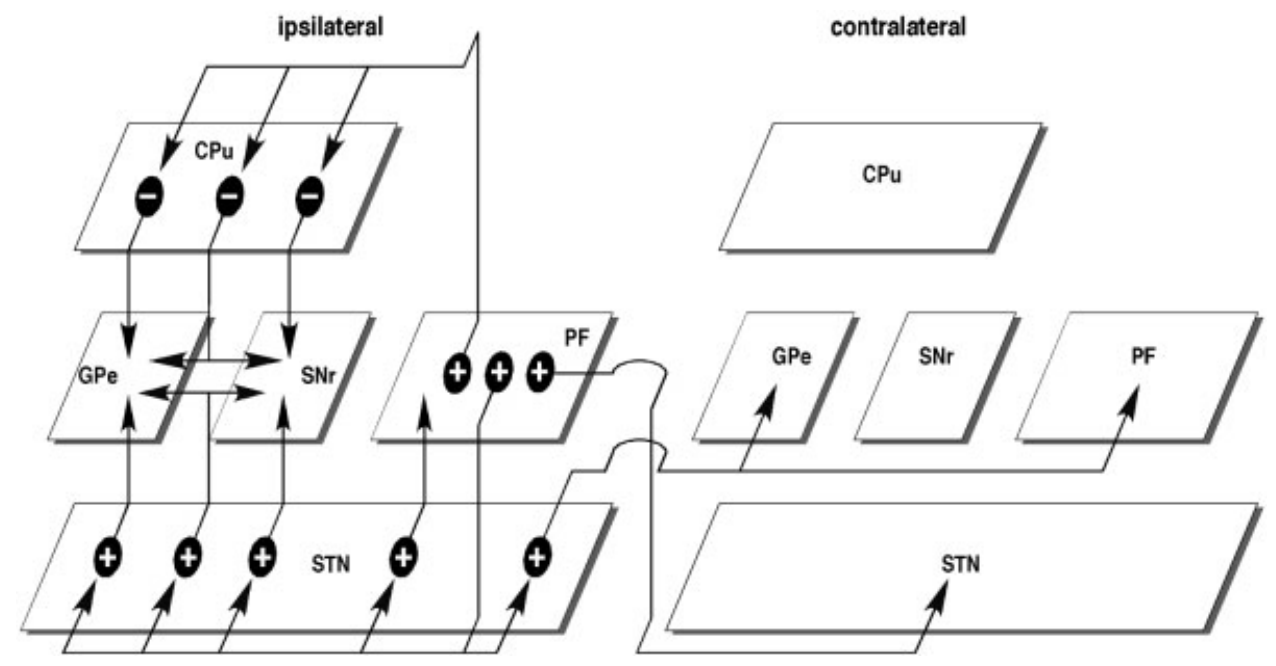

though the precise reason for this overactivity remains to be elucidated. According to Orieux et al. (2000), the metabolic activity of PF neurons projecting to the STN is increased by up to $62 \%$ in dopamine-depleted rats when compared to sham-lesioned animals. In contrast, cortical neurons projecting directly to the STN, the so-called hyperdirect pathway (see Nambu et al., 2002), displayed a marked decrease in the expression of mRNA encoding for subunit I of cytochrome oxidase in 6-OHDA-lesioned rats (Orieux et al., 2002).

There are several pathways that exert both a direct and indirect influence of STN activity through thalamic afferents. First, the STN nucleus receives excitatory, glutamatergic afferents arising from the ipsilateral PF (Sugimoto and Hattori, 1983; Sugimoto et al., 1983; Marini et al., 1999; Gonzalo et al., 2002; Lanciego et al., 2004) as well as from the contralateral $\mathrm{PF}$, the latter being composed of only a few fibers (see also Gerfen et al., 1982). Second, the thalamus might produce indirect excitation of STN neurons by reducing the activity of GP neurons through the thalamostriatal projections to striatopallidal neurons. Finally, it is important to take into account the presence of STN neurons projecting simultaneously to both the contralateral PF and GP, these neurons being consistently found here in all injected cases. A key question is whether the small amount of STN neurons (2-5 neurons per section) projecting contralaterally to both PF and GP is simply an idiosyncrasy of the system or whether it has a significant impact on basal ganglia function. If this were the case, it would be possible to speculate that the activity of one STN nucleus might be under the indirect control of the contralateral STN, since crossed excitatory subthalamopallidal projections could reach GABAergic pallidosubthalamic neurons. In this respect, Mouroux et al. (1995) reported opposing changes recorded in the ipsi- and contralateral STN nucleus after unilateral drug-induced stimulation or inhibition of the PF. The delivery of carbachol into the $\mathrm{PF}$ resulted in a marked increase of ipsilateral STN activity (up to $119 \%$ of excitation) and a decrease in the activity of the contralateral STN (49\%). Opposite effects were noticed after microinjection of muscimol, i.e., inhibition of the ipsilateral STN (91\% of decrease) and excitation of the contralateral STN (47\%). Hence, we con- sider that crossed thalamosubthalamic projections as well as contralateral subthalamopallidal and subthalamothalamic projections might provide the anatomical basis to explain these electrophysiological findings.

There is conclusive evidence that crossed interhemispheric projections at different levels link basal gangliarelated nuclei to several structures located in the contralateral hemisphere. The best characterized of these decussation is the crossed nigrostriatal projection, whose projection constitutes about 1-4\% of the ipsilateral labeling (Fass and Butcher, 1981; Gerfen et al., 1982; Loughlin and Fallon, 1982; Altar et al., 1983; Consolazione et al., 1985; Douglas et al., 1987). The existence of contralateral projections linking the substantia nigra with the thalamus was also demonstrated through projections from the nigral afferents to the mediodorsal thalamic nucleus (Beckstead et al., 1979), as well as to the ventromedial thalamic nucleus (Hekerham, 1979). Contralateral nigrothalamic projections comprise about $5 \%$ of the ipsilateral labeling (Rodríguez et al., 2001). Another decussation is represented by efferents arising from the deep mesencephalic nucleus that reach the contralateral thalamus. Indeed, this crossed projection represents up to $23 \%$ of the ipsilateral projection (Rodríguez et al., 2001; GonzálezHernández et al., 2002). Other sources of contralateral basal ganglia projections involve the bilateral nigral innervation coming from parabrachial-pedunculopontine areas (Gerfen et al., 1982), bilateral nigro-tectal projections (Deniau et al., 1977), descending bilateral projections from PF to the raphe system (Marini and Tredici, 1995), and a small projection linking the STN with the contralateral PF (Gerfen et al., 1982), the latter clearly confirmed by the present data.

\section{CONCLUSION}

Our data are indicative of a key role played by the caudal intralaminar nuclei in basal ganglia organization. Due to the complex arrangement of the ipsi- and contralateral thalamic projections to several basal ganglia nuclei, the caudal intralaminar nuclei can exert a multifaceted influence of both the direct and indirect pathways of basal ganglia transmission at different levels. The data reported 
here, together with that from the literature, call for a reappraisal of the current model of basal ganglia function. First, there is conclusive evidence for a high degree of collateralization in the basal ganglia system, by virtue of which an efferent neuron can simultaneously influence several basal ganglia nuclei. Second, the thalamus can no longer be seen as a simple relay station between the output nuclei and the cortex, since at least the caudal intralaminar nuclei are capable of modulating basal ganglia transmission at different levels. Third, new data suggest the existence of crossed projections arising from $\mathrm{PF}$ and reaching the contralateral STN. Finally, a small population of STN neurons projects simultaneously to both the contralateral GP and PF, via axon collaterals. Although the role of this projection remains to be elucidated, an attractive hypothesis is that the activity of one STN might be under the indirect control of the contralateral STN through crossed subthalamopallidal projections.

\section{LITERATURE CITED}

Albin RL, Young AB, Penney JB. 1989. The functional anatomy of basal ganglia disorders. Trends Neurosci 12:366-375.

Altar A, Neve KA, Loughlin SE, Marshall JF, Fallon JH. 1983. The crossed mesostriatal projection: neurochemistry and developmental response to lesion. Brain Res 279:1-8.

Anderson KD, Reiner A. 1990. Extensive co-occurrence of substance P and dynorphin in striatal projection neurons: an evolutionary conserved feature of basal ganglia organization. J Comp Neurol 295:339-369.

Ariano MA, Fisher RS, Smyk-Randall E, Sibley DR, Levine MS. 1993. D2 dopamine receptor distribution in the rodent CNS using anti-peptide antisera. Brain Res 609:71-80.

Bacci JJ, Kerkerian-Le Goff L, Salin P. 2002. Effects of intralaminar thalamic nuclei lesion on glutamic acid decarboxylase (GAD65 and GAD67) and cytochrome oxidase subunit I mRNA expression in the basal ganglia of the rat. Eur J Neurosci 15:1918-1928.

Bacci JJ, Kachidian P, Kerkerian-Le Goff L, Salin P. 2004. Intralaminar thalamic nuclei lesions: widespread impact on dopamine denervationmediated cellular defects in the rat basal ganglia. J Neuropathol Exp Neurol 63:20-31.

Bar-Gad I, Bergman H. 2001. Stepping out of the box: information processing in the neural networks of the basal ganglia. Curr Opin Neurobiol 11:689-695.

Beckstead RM, Domesik VB, Nauta WJ. 1979. Efferent connections of the substantia nigra and ventral tegmental area in the rat. Brain Res 175:191-217.

Bennet BD, Bolam JP. 1994. Synaptic input and output of parvalbuminimmunoreactive neurons in the neostriatum of the rat. Neuroscience 62:707-719.

Buchwald NA, Price DD, Vernon L, Hull CD. 1973. Caudate intracellular response to thalamic and cortical inputs. Exp Neurol 38:311-323.

Consolazione A, Bentivoglio M, Goldstein M, Toffano G. 1985. Evidence for crossed catecholaminergic nigrostriatal projections by combining wheat germ agglutinin-horseradish peroxidase retrograde transport and tyrosine hydroxylase immunocytochemistry. Brain Res 338:140143.

DeLong MR. 1990. Primate models of movement disorders of basal ganglia origin. Trends Neurosci 13:281-285.

Deniau JM, Hammond-LeGuyader C, Féger J, McKenzie JS. 1977. Bilateral projections of nigro-collicular neurons: an electrophysiological study in the rat. Neurosci Lett 5:45-50.

Deschênes M, Bourassa J, Parent A. 1995. Two different types of thalamic fibers innervate the rat striatum. Brain Res 701:288-292.

Deschênes M, Bourassa J, Doan VD, Parent A. 1996. A single-cell study of the axonal projections arising from the posterior intralaminar thalamic nuclei in the rat. Eur J Neurosci 8:329-343.

Divac I, Mogensen J. 1990. Long-term retrograde labeling of neurons. Brain Res 524:339-341.

Douglas R, Kellaway L, Mintz M, van Wageningen G. 1987. The crossed nigrostriatal projection decussates in the ventral tegmental decussation. Brain Res 418:111-121.
Dubé L, Smith AD, Bolam JP. 1988. Identification of synaptic terminals of thalamic or cortical origin in contact with distinct medium-sized spiny neurons in the rat neostriatum. J Comp Neurol 267:455-471.

Fass B, Butcher LL. 1981. Evidence for a crossed nigrostriatal pathway in rats. Neurosci Lett 22:109-113.

Féger J, Bevan MD, Crossman AR. 1994. The projections from the parafascicular thalamic nucleus to the subthalamic nucleus and striatum arise from separate neuronal populations: a comparison with the corticostriatal and corticosubthalamic efferents in a retrograde double labeling study. Neuroscience 60:125-132.

Gerfen CR. 1992. The neostriatal mosaic: multiple levels of compartmental organization. Trends Neurosci 15:133-139.

Gerfen CR, Staines WA, Arbuthnott GW, Fibiger HC. 1982. Crossed connections of the substantia nigra in the rat. J Comp Neurol 207:283303.

Gerfen CR, Engber TM, Mahan LC, Susel Z, Chase TN, Monsma FJ Jr, Sibley DR. 1990. D1 and D2 dopamine receptor-regulated gene expression of striatonigral and striatopallidal neurons. Science 250:14291432.

González-Hernández T, Barroso-Chinea P, Pérez de la Cruz MA, Valera P, Dopico JG, Rodríguez M. 2002. Response of GABAergic cells in the deep mesencephalic nucleus to dopaminergic cell degeneration: an electrophysiological and in situ hybridization study. Neuroscience 113:311321.

Gonzalo N, Lanciego JL, Castle M, Vázquez A, Erro E, Obeso JA. 2002. The parafascicular thalamic complex and basal ganglia circuitry: further complexity to the basal ganglia model. Thal Rel Sys 1:341-348.

Hamani C, Saint-Cyr JA, Fraser J, Kaplitt M, Lozano A. 2003. The subthalamic nucleus in the context of movement disorders. Brain 127:420.

Hekerham M. 1979. The afferent and efferent connections of the ventromedial thalamic nucleus in the rat. J Comp Neurol 183:487-517.

Henderson JM, Carpenter K, Cartwright H, Halliday GM. 2000a. Degeneration of the centre median-parafascicular complex in Parkinson's disease. Ann Neurol 47:345-352.

Henderson JM, Carpenter K, Cartwright H, Halliday GM. 2000b. Loss of thalamic intralaminar nuclei in progressive supranuclear palsy and Parkinson's disease: clinical and therapeutic implications. Brain 123: 1410-1421.

Inoué S. 1995. Foundations of confocal scanned imaging in light microscopy. In: Pawley JB, editor. Handbook of biological confocal microscopy, 2nd ed. New York: Plenum Press. p 1-17.

Joel D, Weiner I. 1997. The organization of the basal gangliathalamocortical circuits: open interconnected rather than closed segregated. Brain Res Rev 23:62-78.

Kawaguchi Y, Wilson CJ, Emson PC. 1991. Projection subtypes of rat neostriatal matrix cells revealed by intracellular injection of biocytin. J Neurosci 10:3421-3438.

Kincaid AE, Penney JB Jr, Young AB, Newman SW. 1991. The globus pallidus receives a projection from the parafascicular nucleus in the rat. Brain Res 553:18-26.

Kita H, Kitai ST. 1987. Efferent projections of the subthalamic nucleus in the rat: light and electron microscopic analysis with the PHA-L method. J Comp Neurol 260:435-452.

Kita H, Chang HT, Kitai ST. 1983. The morphology of intracellularly labeled rat subthalamic neurons: a light microscopic analysis. J Comp Neurol 215:245-257.

Kitai ST, Kocsis JD, Preston RJ, Sugimori M. 1976. Monosynaptic inputs to caudate neurons identified by intracellular injection of horseradish peroxidase. Brain Res 109:601-606.

Kocsis JD, Sugimori M, Kitai ST. 1977. Convergence of excitatory synaptic inputs to caudate spiny neurons. Brain Res 124:403-413.

Lanciego JL, Gonzalo N, Castle M, Sánchez-Escobar C, Aymerich MS, Obeso JA. 2004. Thalamic innervation of striatal and subthalamic neurons projecting to the rat entopeduncular nucleus. Eur J Neurosci 19:1267-1277.

Lapper SR, Bolam JP. 1992. Input from the frontal cortex and the parafascicular nucleus to cholinergic interneurons in the dorsal striatum of the rat. Neuroscience 51:533-545.

Le Moine C, Normand E, Bloch B. 1991. Phenotypical characterization of the rat striatal neurons expressing the D1 dopamine receptor gene. Proc Natl Acad Sci U S A 88:4205-4209.

Loughlin SE, Fallon JH. 1982. Mesostriatal projections from ventral tegmentum and dorsal raphe: cells project ipsilaterally or contralaterally but not bilaterally. Neurosci Lett 32:11-16. 
Marini G, Tredici G. 1995. Parafascicular nucleus-raphe projections and termination patterns in the rat. Brain Res 690:177-184.

Marini G, Pianca L, Tredici G. 1999. Descending projections arising from the parafascicular nucleus in rats. Trajectory of fibers, projection patter and mapping of terminations. Somatosens Motor Res 16:207-222.

Meador-Woodruff JH, Mansour A, Healy DJ, Kuehn R, Zhou QY, Bunzow JR, Akil H, Civelli O, Watson SJ Jr. 1991. Comparison of the distribution of D1 and D2 dopamine receptor mRNAs in rat brain. Neuropsychopharmacology 5:231-242.

Mouroux M, Féger J. 1993. Evidence that the parafascicular projection to the subthalamic nucleus is glutamatergic. NeuroReport 4:613-615.

Mouroux M, Hassani OK, Féger J. 1995. Electrophysiological study of the excitatory parafascicular projection to the subthalamic nucleus and evidence for ipsi- and contralateral controls. Neuroscience 67:399-407.

Nambu A. 2004. A new dynamic model of the cortico-basal ganglia loop. Prog Brain Res 143:461-466.

Nambu A, Tokuno H, Takada M. 2002. Functional significance of the cortico-subthalamo-pallidal 'hyperdirect' pathway. Neurosci Res 43: 111-117.

Obeso JA, Rodríguez-Oroz MC, Rodríguez M, Lanciego JL, Artieda J, Gonzalo N, Olanow CW. 2000. Pathophysiology of the basal ganglia in Parkinson's disease. Trends Neurosci 23:S8-S19.

Orieux G, François C, Féger J, Yelnik J, Vila M, Ruberg M, Agid Y, Hirsch EC. 2000. Metabolic activity of excitatory parafascicular and pedunculopontine inputs to the subthalamic nucleus in a rat model of Parkinson's disease. Neuroscience 97:79-88.

Orieux G, François C, Féger J, Hirsch EC. 2002. Consequences of dopaminergic denervation on the metabolic activity of the cortical neurons projecting to the subthalamic nucleus in the rat. J Neurosci 22:87628770 .

Parent A, Charara A, Pinault D. 1995. Single striatofugal axons arborizing in both pallidal segments and in the substantia nigra in primates. Brain Res 698:280-284.

Parent A, Sato F, Wu Y, Gauthier J, Lévesque M, Parent M. 2000. Organization of the basal ganglia: the importance of axonal collateralization. Trends Neurosci 23:S20-S27.

Pawley JB. 1995. Fundamental limits in confocal microscopy. In: Pawley JB, editor. Handbook of biological confocal microscopy, 2nd ed. New York: Plenum Press. p 1-17.

Paxinos G, Watson C. 1998. Atlas of the rat brain in stereotaxic coordinates, 4th ed. New York: Academic Press.

Pieribone VA, Aston-Jones G. 1988. The iontophoretic application of Fluoro-Gold for the study of afferents to deep brain nuclei. Brain Res 475:259-271.

Plenz D, Kitai ST. 1999. A basal ganglia pacemaker formed by the subthalamic nucleus and external globus pallidus. Nature 400:677-682.

Reiner A, Anderson KD. 1990. The patterns of neurostransmitter and neuropeptide co-occurrence among striatal projection neurons: conclusions based on recent findings. Brain Res Rev 15:251-265.

Robertson HA. 1992. Dopamine receptor interactions: some implications for the treatment of Parkinson's disease. Trends Neurosci 15:201-206.

Rodríguez M, Abdala P, Barroso-Chinea P, González-Hernández T. 2001. The deep mesencephalic nucleus as an output center of basal ganglia: morphological and electrophysiological similarities with the substantia nigra. J Comp Neurol 438:12-31.

Rosene DL, Roy NJ, Davis BJ. 1986. A cryoprotection method that facilitates cutting frozen sections of whole monkey brain for histological and histochemical processing without freezing artifact. J Histochem Cytochem 34:1301-1316.
Rudkin TM, Sadikot AF. 1999. Thalamic input to parvalbuminimmunoreactive GABAergic interneurons: organization in normal striatum and effect on neonatal decortication. Neuroscience 88:1165-1175.

Sato F, Parent M, Levesque M, Parent A. 2000. Axonal branching pattern of neurons of the subthalamic nucleus in primates. J Comp Neurol 424:142-152.

Schmued LC, Fallon JH. 1986. Fluoro-Gold: a new fluorescent retrograde axonal tracer with numerous unique properties. Brain Res 377:147154.

Schmued LC, Heimer L. 1990. Iontophoretic injection of Fluoro-Gold and other fluorescent tracers. J Histochem Cytochem 38:721-723.

Shink E, Bevan MD, Bolam JP, Smith Y. 1996. The subthalamic nucleus and the external globus pallidum: two tightly interconnected structures that control the output of the basal ganglia in the monkey. Neuroscience 73:335-357.

Sidibé M, Smith Y. 1996. Differential synaptic innervation of striatofugal neurons projecting to the internal or external segments of the globus pallidus by thalamic afferents in the squirrel monkey. J Comp Neurol 365:445-465.

Sidibé M, Smith Y. 1999. Thalamic input to striatal interneurons in monkeys: synaptic organization and co-localization of calcium binding proteins. Neuroscience 89:1189-1208.

Sugimoto T, Hattori T. 1983. Confirmation of the thalamosubthalamic projection by electron microscope autoradiography. Brain Res 267:335339 .

Sugimoto T, Hattori T, Mizuno N, Itoh K, Sato M. 1983. Direct projections from the centromedian-parafascicular complex to the subthalamic nucleus in the cat and rat. J Comp Neurol 214:209-216.

Surmeier DJ, Eberwine J, Wilson CJ, Cao Y, Stefani A, Kitai ST. 1992. Dopamine receptor subtypes colocalize in rat striatonigral neurons. Proc Natl Acad Sci U S A 89:10178-10182.

Surmeier DJ, Reiner A, Levine MS, Ariano MA. 1993. Are neostriatal dopamine receptors co-localized? Trends Neurosci 16:299-305.

Van der Kooy D, Hattori T. 1980. Single subthalamic nucleus neurons project to both the globus pallidus and substantia nigra in rat. J Comp Neurol 192:751-768.

Vercelli A, Marini G, Tredici G. 2003. Anatomical organization of the telencephalic connections of the parafascicular nucleus in adult and developing rats. Eur J Neurosci 18:275-289.

Weiner DM, Levey AI, Sunahara RK, Niznik HB, O'Dowd BF, Seeman P Brann MR. 1991. D1 and D2 dopamine receptor mRNA in rat brain. Proc Natl Acad Sci U S A 88:1859-1863.

Wilson CJ, Chang HT, Kitai ST. 1983. Origins of postsynaptic potentials evoked in spiny neostriatal projection neurons by thalamic stimulation in the rat. Exp Brain Res 51:217-226.

Wouterlood FG, Vinkenoog M, van den Oever M. 2002a. Tracing tools to resolve neural circuits. Network 13:327-342.

Wouterlood FG, van Haeften T, Blijleven N, Pérez-Templado P, PérezTemplado H. 2002b. Double-label confocal laser-scanning microscopy, image restoration, and real-time three-dimensional reconstruction to study axons in the central nervous system and their contacts with target neurons. Appl Immunohistochem Mol Morphol 10:85-95.

Wu Y, Richard S, Parent A. 2000. The organization of the striatal output system: a single-cell juxtacellular labeling study in the rat. Neurosci Res 38:49-62.

Yasukawa T, Kita T, Xue Y, Kita H. 2004. Rat intralaminar thalamic nuclei projections to the globus pallidus: a biotinylated dextran amine anterograde tracing study. J Comp Neurol 471:153-167. 